\title{
Measuring and Maximizing Influence via Random Walk in Social Activity Networks
}

\author{
Pengpeng Zhao ${ }^{1}$, Yongkun $\mathrm{Li}^{1,2(凶)}$, Hong Xie ${ }^{3}$, Zhiyong $\mathrm{Wu}^{1}$ \\ Yinlong $\mathrm{Xu}^{1,4}$, John C. S. Lui ${ }^{5}$ \\ ${ }^{1}$ University of Science and Technology of China, Hefei, China \\ \{roczhau, wzylucky\}@mail.ustc.edu.cn, \{ykli,ylxu\}@ustc.edu.cn \\ ${ }^{2}$ Collaborative Innovation Center of High Performance Computing, National University of \\ Defense Technology, Changsha, China \\ ${ }^{3}$ National University of Singapore, Singapore, Singapore \\ hongx87@gmail.com \\ ${ }^{4}$ AnHui Province Key Laboratory of High Performance Computing, Hefei, China \\ ${ }^{5}$ The Chinese University of Hong Kong, Hong Kong, China \\ cslui@cse.cuhk.edu.hk
}

\begin{abstract}
With the popularity of OSNs, finding a set of most influential users (or nodes) so as to trigger the largest influence cascade is of significance. For example, companies may take advantage of the "word-of-mouth" effect to trigger a large cascade of purchases by offering free samples/discounts to those most influential users. This task is usually modeled as an influence maximization problem, and it has been widely studied in the past decade. However, considering that users in OSNs may participate in various kinds of online activities, e.g., giving ratings to products, joining discussion groups, etc., influence diffusion through online activities becomes even more significant.

In this paper, we study the impact of online activities by formulating the influence maximization problem for social-activity networks (SANs) containing both users and online activities. To address the computation challenge, we define an influence centrality via random walks to measure influence, then use the Monte Carlo framework to efficiently estimate the centrality in SANs. Furthermore, we develop a greedy-based algorithm with two novel optimization techniques to find the most influential users. By conducting extensive experiments with real-world datasets, we show our approach is more efficient than the state-of-the-art algorithm IMM [17] when we needs to handle large amount of online activities.
\end{abstract}

Keywords: OSN, Influence Maximization, Random Walk

\section{Introduction}

Due to the popularity of online social networks (OSNs), viral marketing which exploits the "word-of-mouth" effect is of significance to companies which want to promote product sales. Therefore, it is of interest to find the best initial set of users so as to trigger the largest influence spread. This viral marking problem can be modeled as an influence maximization problem, which was first formulated by Kempe et al. [12]. That is, given an OSN and an information diffusion model, how to select a set of $k$ users, 


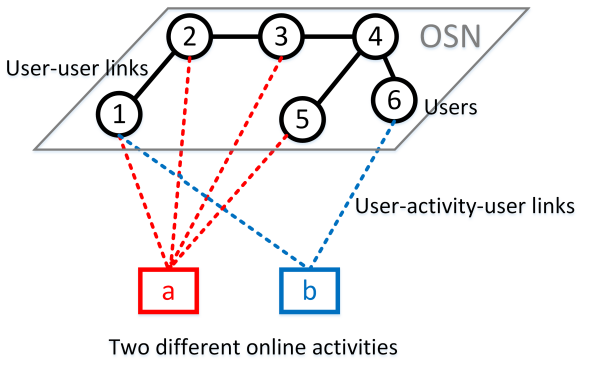

Fig. 1: An example of social-activity network (SAN).

which is called the seed set, so as to trigger the largest influence spread. This problem is proved to be an NP-hard problem [4, 6], and it has been studied extensively in the past decade [4-6, 17, 18].

Note that users in today's OSNs may participate in various kinds of online activities, e.g., joining a discussion group, and clicking like on Facebook etc. Hence, users not only can create friendship relationships, which we call user-user links, but can also form relationships by participating in online activities, which we call user-activity-user links. For example, if two users in Facebook express like to the same public page, then they form a user-activity-user link no matter they are friends or not. We call this kind of networks which contain both user-user relationships and user-activity-user relationships as social-activity networks (SANs).

With the consideration of online activities in SANs, influence may also spread through the user-activity-user links as well as the user-user links. In this paper, we focus on the online activities which generate positive influence, e.g., clicking like on the same public page in Facebook, giving high rating to the same product in online rating systems, and joining in a community sharing the same interest in online social networks. Due to the large amount of online activities, e.g., each pair of users may participate in multiple online activities, influence diffusion through the user-activity-user links becomes even more significant, and so only considering OSNs alone may not trigger the largest influence spread. Existing works on influence maximization usually focus on OSNs only and do not take the impact of online activities into consideration. This motivates us to formulate the influence maximization problem for SANs, and to determine the most influential nodes by taking online activities into consideration.

However, solving the influence maximization problem in SANs with online activities is challenging. First, influence maximization in OSNs without online activities was already proved to be NP-hard, and considering online activities makes this problem even more complicated. Second, the amount of online activities in a SAN is very large even for small OSNs, this is because online activities happen more frequently than friendship formation in OSNs. As a result, the underlying graph which characterizes users and their relationships may become extremely dense if we transform the user-activity-user links to user-user links, so it requires highly efficient algorithms for finding the most influential nodes. To address the above challenges, in this paper, we make the following contributions. 
- We generalize the influence diffusion models for SANs by modeling SANs as hypergraphs, and approximate the influence of nodes in SANs by defining an influence centrality based on random walk.

- We employ the Monte Carlo framework to estimate the influence centrality in SANs, and also develop a greedy-based algorithm with two novel optimization techniques to solve the influence maximization problem for SANs.

- We conduct experiments with real-world datasets, and results show that our approach is more efficient while keep almost the same accuracy compared to the state-of-the-art algorithm.

This paper is organized as follows. In $\$ 2$, we formulate the influence maximization problem for SANs. In $\$ 3$ we present our random walk based methodology. In $\$$, we present the Monte Carlo method to estimate the influence centrality in SANs. In 5 , we present our greedy-based algorithm and optimization techniques to solve the influence maximization problem. In $\$$ we present the experimental results. Related work is given in $\$ 7$ and 88 concludes.

\section{Problem Formulation}

In this section, we first model the SAN with a hypergraph, and then formulate the influence maximization problem for SANs.

\subsection{Model for SANs}

We use a hypergraph $G\left(V, E, \mathcal{E}_{1}, \ldots, \mathcal{E}_{l}\right)$ to characterize a SAN, where $V$ denotes the set of users, $E$ denotes the user-user links, and $\mathcal{E}_{i}(i=1,2, \ldots, l)$ denotes the set of type $i$ hyperedges in which each hyperedge is a set of users who participated in the same online activity, and represented as a tuple. Considering Figure 1, only activity $a$ is of the first type, so $\mathcal{E}_{1}=\{(1,2,3,5)\}$. For ease of presentation, we denote $N(j)$ as the set of neighbors of user $j$, i.e., $N(j)=\{i \mid(i, j) \in E\}, M_{e}(j)$ as the set of users except for user $j$ who connected to the hyperedge $e$, i.e., $M_{e}(j)=\{i \mid i \in e, \& i \neq$ $j\}$, and denote $\mathcal{E}_{t}(j)$ as the set of type $t$ hyperedges that are connected to user $j$, i.e., $\mathcal{E}_{t}(j)=\left\{e \mid e \in \mathcal{E}_{t} \& j \in e\right\}$. Considering Figure $10(1)=\{2\}, M_{e}(1)=\{2,3,5\}$ when $e=(1,2,3,5)$ and $\mathcal{E}_{1}(1)=\{(1,2,3,5)\}$.

\subsection{Influence Maximization in SANs}

Before describe the influence diffusion process for SANs, we first recall the independent cascade model(IC) which was proposed by Kempe et al. in [12]. Suppose that each user has two states, either active or inactive. At first, we initialize a set of users as active. For an active user $i$, she will activate each of her inactive neighbor $j(j \in N(i)$ where $N(i)$ denotes the neighbor set of user $i)$ with probability $q_{i j}\left(0 \leq q_{i j} \leq 1\right)$. One common setting of $q_{i j}$ is $q_{i j}=\frac{1}{d_{j}}$, e.g., [4, 5, 12, 17, 18], where $d_{j}$ denotes the degree of user $j$, i.e., $d_{j}=|N(j)|$. After a neighbor $j$ being activated, then she will further activate her inactive neighbors in the set $N(j)$, and this diffusion process continues until no user can 
change her state. We call the expected size of the final set of active users the influence spread, and denote it as $\sigma(S(k))$ if the set of $k$ initial active users is $S(k)$.

Now we describe the influence diffusion process for SANs. The key issue is to define the influence between user $i$ and user $j$ (i.e., $g_{i j}$ ) after taking online activities into consideration. Our definition is based on three criteria:

- A user may make a purchase due to her own interest or being influenced by others through user-user or user-activity-user links, so we define the total influence probability by one-hop neighbors as $c(0<c<1)$, and call it the decay parameter. As we have $l$ types of online activities, we define $\alpha_{j t}$ (where $0 \leq \alpha_{j t} \leq 1$ and $0 \leq \sum_{t=1}^{l} \alpha_{j t} \leq 1$ ) as the proportion of influence to user $j$ through type $t$ online activities, and call it weight of activities. Clearly, $1-\sum_{t=1}^{l} \alpha_{j t}$ indicates the proportion of influence from direct neighbors.

- For the influence to user $j$ from direct neighbors, we define the weight of each neighbor $i(i \in N(j))$ as $u_{i j}$, and assume that $0 \leq u_{i j} \leq 1$ and $\sum_{i \in N(j)} u_{i j}=1$.

- For the influence to user $j$ through the type $t$ online activities, we define the weight of each online activity $a$ as $v_{a j}$, where $0 \leq v_{a j} \leq 1$ and $\sum_{a \in N_{t}(j)} v_{a j}=1$. Besides, considering that maybe multiple users participated in the same online activity $a$, we define the weight of each user $i$ who participated in $a$ as $u_{i j}^{a}(i \in N(a) \backslash\{j\})$, and assume that $0 \leq u_{i j}^{a} \leq 1$ and $\sum_{i \in N(a) \backslash\{j\}} u_{i j}^{a}=1$.

For simplicity, we let $u_{i j}=1 /|N(j)|$ in this paper. Note that this uniform setting is exactly the same as the IC model in OSNs, which has been widely studied in [4, 5, 12, 17, 18]. Similarly, we also let $v_{a j}=1 /\left|\mathcal{E}_{t}(j)\right|$ and $u_{i j}^{a}=1 /\left|M_{e}(j)\right|$ by following the uniform setting. We would like to point out that our random walk approach in this paper also applies to general settings. Now we can define the influence of user $i$ to user $j$, which we denote as $g_{i j}$ :

$$
g_{i j}=c \times\left(\frac{1-\sum_{t=1}^{l} \alpha_{j t}}{|N(j)|} \times \mathbf{1}_{\{i \in N(j)\}}+\sum_{t \in[1, l]} \sum_{e \in \mathcal{E}_{t}(j)} \frac{\alpha_{j t}}{\left|\mathcal{E}_{t}(j)\right|} \times \frac{1}{\left|M_{e}(j)\right|} \times \mathbf{1}_{\left\{i \in M_{e}(j)\right\}}\right) .
$$

The first part in the right hand side of Equation (1) denotes the influence diffusion through user-user links, and the second part represents the influence diffusion through user-activity-user links.

Now we formulate the influence maximization problem for SANs, which we denote as IMP(SAN), as follows.

Definition 1. IMP(SAN): Given a SAN $G\left(V, E, \mathcal{E}_{1}, \ldots, \mathcal{E}_{l}\right)$, an influence diffusion model with parameters $\alpha_{j t}$, find a set of $k$ nodes $S(k)$, where $k$ is an integer, so as to make the influence spread $\sigma(S(k))$ maximized.

\section{Methodology}

In this section, we present our methodology to address the (IMP(SAN)) problem. To reduce the large computation cost, we first develop a random walk framework on hypergraphs to estimate the influence diffusion process. Then, we define a centrality measure based on random walk to approximate the influence of a node set. With this centrality measure, we can approximate the influence maximization problem by solving a centrality maximization problem. 


\subsection{Random Walk on Hypergraph}

Here, we present our random walk based framework, which is extended from the classical random walk on a simple unweighted graph $G(V, E)$, which can be stated as follows. For a random walk at vertex $i \in V$, it uniformly selects at random a neighbor $j$ ( $j \in N(i)$ ), and then moves to $j$ in the next step. Mathematically, if we denote $Y(t)$ as the position of the walker at step $t$, then $\{Y(t)\}$ constitutes a Markov chain where the one-step transition probability $p_{i j}$ is defined as $p_{i j}=1 /|N(i)|$ if $(i, j) \in E$, and 0 otherwise.

We now define the one-step transition probability $p_{i j}$ when performing a random walk on the hypergraph $G\left(V, E, \mathcal{E}_{1}, \ldots, \mathcal{E}_{l}\right)$. Note that each hyperedge may contain more than two vertices, so we take the one-step random walk from user $i$ to user $j$ as a twostep process.

- Step one: Choose a hyperedge associated to user $i$. Precisely, according to the influence diffusion models in 2.2 we set the probability of selecting type $t$ hyperedges as $\alpha_{i t}$, and choose hyperedges of the same type uniformly at random. Mathematically, if the walker is currently at user $i$, then it chooses a hyperedge $e$ of type $t$ with probability $\frac{\alpha_{i t}}{\left|\mathcal{E}_{t}(i)\right|}$.

- Step two: Choose a user associated to the hyperedge $e$ selected in step one as the next stop of the random walk. We consider random walks without backtrace. In particular, if a walker is currently at node $i$, then we select the next stop uniformly from the vertices that are connected to the same hyperedge with user $i$. We define the probability of choosing user $j$ as $1 /\left|M_{e}(i)\right|$.

By combing the two steps defined above, we can derive the transition probability from user $i$ to $j$ as follows, and we can find $g_{j i}=c \times p_{i j}$.

$$
p_{i j}=\frac{1-\sum_{t=1}^{l} \alpha_{i t}}{|N(i)|} \times \mathbf{1}_{\{j \in N(i)\}}+\sum_{t \in[1, l]} \sum_{e \in \mathcal{E}_{t}(i)} \frac{\alpha_{i t}}{\left|\mathcal{E}_{t}(i)\right|} \times \frac{1}{\left|M_{e}(i)\right|} \times \mathbf{1}_{\left\{j \in M_{e}(i)\right\}} .
$$

\subsection{Influence Centrality Measure}

To address the IMP(SAN) problem, one key issue is to measure the influence of a node set. To achieve this, we define a centrality measure based on random walks on hypergraphs to approximate the influence of a node set $S$. We call it influence centrality, and denote it as $I(S)$, which is defined as follows.

$$
I(S)=\sum_{j \in V} h(j, S)
$$

where $h(j, S)$ aims to approximate the influence of $S$ to $j$, which is called decayed hitting probability. It is defined as

$$
h(j, S)=\left\{\begin{array}{l}
\sum_{i \in V} c p_{j i} h(i, S), j \notin S \\
1, j \in S
\end{array}\right.
$$

where $c$ is the decay parameter defined in $\$ 2.2$ and $p_{j i}$ is the one-step transition probability defined in Equation (2). 
To solve the influence maximization problem of IMP(SAN), we use the influence centrality measure $I(S)$ to approximate the influence of the node set $S$, and our goal is to find a set $S$ of $k$ users so that $I(S)$ is maximized. In other words, we approximate the influence maximization problem IMP(SAN) by solving the centrality maximization problem CMP defined as follows.

Definition 2. CMP: Given a hyperghraph $G\left(V, E, \mathcal{E}_{1}, \ldots, \mathcal{E}_{l}\right)$ and the corresponding parameters $\alpha_{j t}$, find a set $S$ of $k$ nodes, where $k$ is an integer, so as to make the influence centrality of the set $S$ of $k$ nodes $I(S)$ maximized.

\section{Centrality Computation}

We note that the key challenge of solving the centrality maximization problem CMP is how to efficiently estimate the influence centrality of a node set $I(S)$, or the decayed hitting probability $h(j, S)$. We give an efficient framework to estimate $h(j, S)$ as follows. We first rewrite $h(j, S)$ in a linear expression which is an infinite converging series, and then truncate the converging series to save computation time (see 4.1 . To further estimate the truncated series, we first explain the expression with a random walk approach, and then use a Monte Carlo framework via random walks to estimate it efficiently (see 4.2 .

\subsection{Linear Expression}

We first transform $h(j, S)$ defined in Equation (4) to a linear expression.

Theorem 1. The decayed hitting probability $h(j, S)$ can be rewritten as

$$
h(j, S)=c \boldsymbol{e}_{j}^{T} \boldsymbol{Q}^{\prime} \boldsymbol{e}+c^{2} \boldsymbol{e}_{j}^{T} \boldsymbol{Q} \boldsymbol{Q}^{\prime} \boldsymbol{e}+c^{3} \boldsymbol{e}_{j}^{T} \boldsymbol{Q}^{2} \boldsymbol{Q}^{\prime} \boldsymbol{e}+\cdots .
$$

where $Q$ is a $(|V|-|S|) \times(|V|-|S|)$ dimensional matrix which describes the transition probabilities between two nodes in the set $V-S, Q^{\prime}$ is a $(|V|-|S|) \times|S|$ dimensional matrix which describes the transition probabilities from a node in $V-S$ to a node in $S, \boldsymbol{I}$ is an identity matrix, $\boldsymbol{e}$ is a column vector with all elements being 1 , and finally $\boldsymbol{e}_{j}$ is a column vector with only the element corresponding to node $j$ being 1 and 0 for all other elements.

Proof: Please refer to the Appendix.

We only keep the $L$ leading terms of the infinite series, and denote the truncated result as $h^{L}(j, S)$, so we have

$$
h^{L}(j, S)=c \boldsymbol{e}_{j}^{T} \boldsymbol{Q}^{\prime} \boldsymbol{e}+c^{2} \boldsymbol{e}_{j}^{T} \boldsymbol{Q} \boldsymbol{Q}^{\prime} \boldsymbol{e}+\ldots+c^{L} \boldsymbol{e}_{j}^{T} \boldsymbol{Q}^{L-1} \boldsymbol{Q}^{\prime} \boldsymbol{e} .
$$

Since $c$ is defined as $0<c<1$, the series truncation error is bounded as follows.

$$
0 \leq h(j, S)-h^{L}(j, S) \leq c^{L+1} /(1-c) .
$$

Based on the above error bound, we can see that $h^{L}(j, S)$ converges to $h(j, S)$ with rate $c^{L+1}$. This implies that if we want to compute $h(j, S)$ with a maximum error $\epsilon$ ( $0 \leq \epsilon \leq 1)$, we only need to compute $h^{L}(j, S)$ by taking a sufficiently large enough $L$, or $L \geq\left\lceil\frac{\log (\epsilon-\epsilon c)}{\log c}\right\rceil-1$. 


\subsection{Monte Carlo Algorithm}

In this subsection, we present a Monte Carlo algorithm to efficiently approximate $h^{L}(j, S)$. Our algorithm is inspired from the random walk interpretation of Equation (6), and it can achieve a high accuracy with a small number of walks.

Consider the random walk interpretation of a particular term $\boldsymbol{e}_{j}^{T} \boldsymbol{Q}^{t-1} \boldsymbol{Q}^{\prime} \boldsymbol{e}(t=$ $1, \ldots, L)$ in Equation (6). Let us consider a $L$-step random walk starting from $j \notin S$ on the hypergraph. At each step, if the walker is currently at node $k(k \notin S)$, then it selects a node $i$ and transits to $i$ with probability $p_{k i}$, which is defined in Equation (2). As long as the walker hits a node in $S$, then it stops. Let $j^{(t)}$ be the $t$-th step position, and define an indicator $X(t)$ as

$$
X(t)= \begin{cases}1, & j^{(t)} \in S, \\ 0, & j^{(t)} \notin S .\end{cases}
$$

We can see that $\boldsymbol{e}_{j}^{T} \boldsymbol{Q}^{t-1} \boldsymbol{Q}^{\prime} \boldsymbol{e}$ is the probability that a random walk starting from $j$ hits a node in $S$ at the $t$-th step. We have

$$
\boldsymbol{e}_{j}^{T} \boldsymbol{Q}^{t-1} \boldsymbol{Q}^{\prime} \boldsymbol{e}=E[X(t)] .
$$

By substituting $\boldsymbol{e}_{j}^{T} \boldsymbol{Q}^{t-1} \boldsymbol{Q}^{\prime} \boldsymbol{e}$ with Equation $\mathbb{8}$, we can rewrite $h^{L}(j, S)$ as

$$
h^{L}(j, S)=c E[X(1)]+c^{2} E[X(2)]+\cdots+c^{L} E[X(L)] .
$$

Now we estimate $h^{L}(j, S)$ by using a Monte Carlo method with random walks on the hypergraph based on Equation (96). Specifically, for each node $j$ where $j \notin S$, we set $R$ independent $L$-step random walks starting from $j$. We denote the $t$-th step position of the $R$ random walks as $j_{1}^{(t)}, j_{2}^{(t)}, \ldots, j_{R}^{(t)}$, respectively, and use $X_{r}(t)$ to indicate whether $j_{r}^{(t)}$ belongs to set $S$ or not. Precisely, we set $X_{r}(t)=1$ if $j_{r}^{(t)} \in S$, and 0 otherwise, so $c^{t} E[X(t)]$ can be estimated as

$$
c^{t} E[X(t)] \approx \frac{c^{t}}{R} \sum_{r=1}^{R} X_{r}(t) .
$$

By substituting $c^{t} E[X(t)]$ in Equation (9), we can approximate $h^{L}(j, S)$, which we denote as $\hat{h}^{L}(j, S)$, as follows.

$$
\hat{h}^{L}(j, S)=\frac{c}{R} \sum_{r=1}^{R} X_{r}(1)+\cdots+\frac{c^{L}}{R} \sum_{r=1}^{R} X_{r}(L) .
$$

Algorithm 1 presents the process of the Monte Carlo method described above. We can see that its time complexity is $O(R L)$ as the number of types of online activities $l$ is usually a small number. In other words, we can estimate $h^{L}(j, S)$ in $O(R L)$ time and compute $I(S)$ in $O(n R L)$ time as we need to estimate $h^{L}(j, S)$ for all nodes. The main benefit of this Monte Carlo algorithm is that its running time is independent of the graph size, so it scales well to large graphs.

Note that $\hat{h}^{L}(j, S)$ computed with Algorithm 1 is an approximation of $h^{L}(j, S)$, and the approximation error depends on the sample size $R$. To estimate the number of samples required to compute $h^{L}(j, S)$ accurately, we derive the error bound by applying Hoeffding inequality [10], and the results are as follows. 


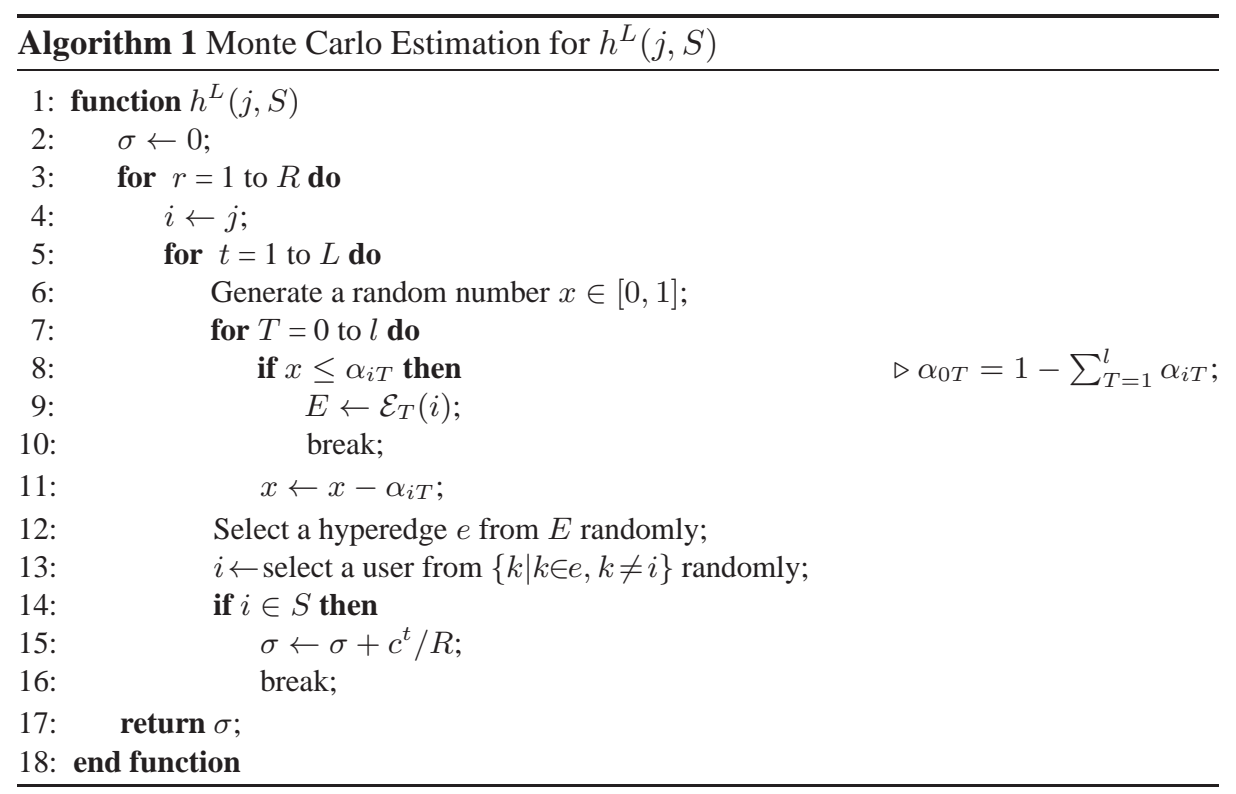

Theorem 2. Let the output of Algorithm $\square$ be $\hat{h}^{L}(j, S)$, then we have

$$
P\left\{\left|\hat{h}^{L}(j, S)-h^{L}(j, S)\right|>\epsilon\right\} \leq 2 L \exp \left(-2(1-c)^{2} \epsilon^{2} R\right) .
$$

Proof: Please refer to the Appendix.

Based on Theorem 2, we see that Algorithm 1 can estimate $h^{L}(j, S)$ with a maximum error $\epsilon$ with least probability $1-\delta(0<\delta, \epsilon<1)$ by setting $R \geq \log (2 L / \delta) /(2(1-$ $c)^{2} \epsilon^{2}$.

\section{Centrality Maximization}

In this section, we develop efficient algorithms to address the centrality maximization problem CMP defined in 3.2 . Noted that even though we can efficiently estimate the decayed hitting probability $h(j, S)$ by using random walks (see 44 , finding a set $S$ of $k$ nodes in a SAN to maximize its influence centrality $I(S)$ is still computationally difficult as it requires to estimate the influence centrality of all combinations of $k$ nodes. In particular, CMP is NP-hard.

Theorem 3. The centrality maximization problem CMP is NP-hard.

Proof: Please refer to the Appendix.

To solve the centrality maximization problem CMP, we develop greedy-based approximation algorithms by exploiting the submodularity property of $I(S)$. Specifically, we first show the submodularity property and present a baseline greedy algorithm to maximize $I(S)$, and then develop two novel optimization techniques to accelerate the greedy algorithm. 


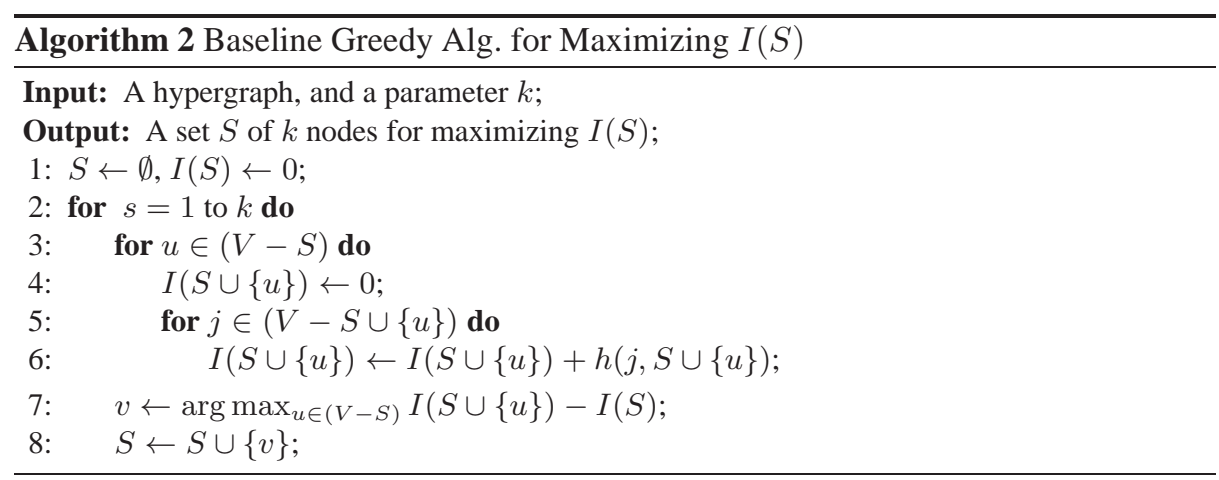

\subsection{Baseline Greedy Algorithm}

Before presenting the greedy-based approximation algorithm for maximizing $I(S)$, we first show that $I(S)$ is a non-decreasing submodular function, and the result is stated in the following theorem.

Theorem 4. The centrality $I(S)$ is a non-decreasing submodular function.

Proof: Please refer to the Appendix.

Based on the submodularity property, we develop a greedy algorithm for approximation when maximizing $I(S)$, and we call it the baseline greedy algorithm. Algorithm 2 describes this procedure. To find a set of $k$ nodes to maximize $I(S)$, the algorithm works for $k$ iterations. In each iteration, it selects the node which maximizes the increment of $I(S)$.

Recall that the time complexity for estimating the influence of a set $S$ to a particular node $j \notin S$, i.e., $h(j, S)$, is $O(R L)$ (see 4.2 ). Thus, the total time complexity for the baseline greedy algorithm is $O\left(k n^{2} R L\right)$ where $n$ denotes the total number of users in the SAN, because estimating the influence of a set $S \cup\{u\}$ requires us to sum up its influence to all nodes, and we need to check every node $u$ so as to select the one which maximizes the increment of $I(S)$. Although the baseline greedy algorithm gives a polynomial time complexity, it is inefficient when the number of users becomes large. To further speed up the computation, we present two novel optimization techniques in the next subsection.

\subsection{Optimizations}

- Parallel Computation: The key component in the greedy algorithm is to measure the marginal increment of the influence after adding node $u$, i.e., $\Delta(u)=I(S \cup\{u\})-I(S)$, which can be derived as follows.

$\Delta(u)=\left[1-\sum_{h=1}^{\infty} c^{h} P(u, S, h)\right] \times\left[1+\sum_{j \in(V-S \cup\{u\})} \sum_{h=1}^{\infty} c^{h} P^{S}(j,\{u\}, h)\right]$.

In the baseline greedy algorithm, $\Delta(u)$ 's are computed sequentially, which as a result incurs a large time overhead. Our main idea to speed up the computation is to estimate 

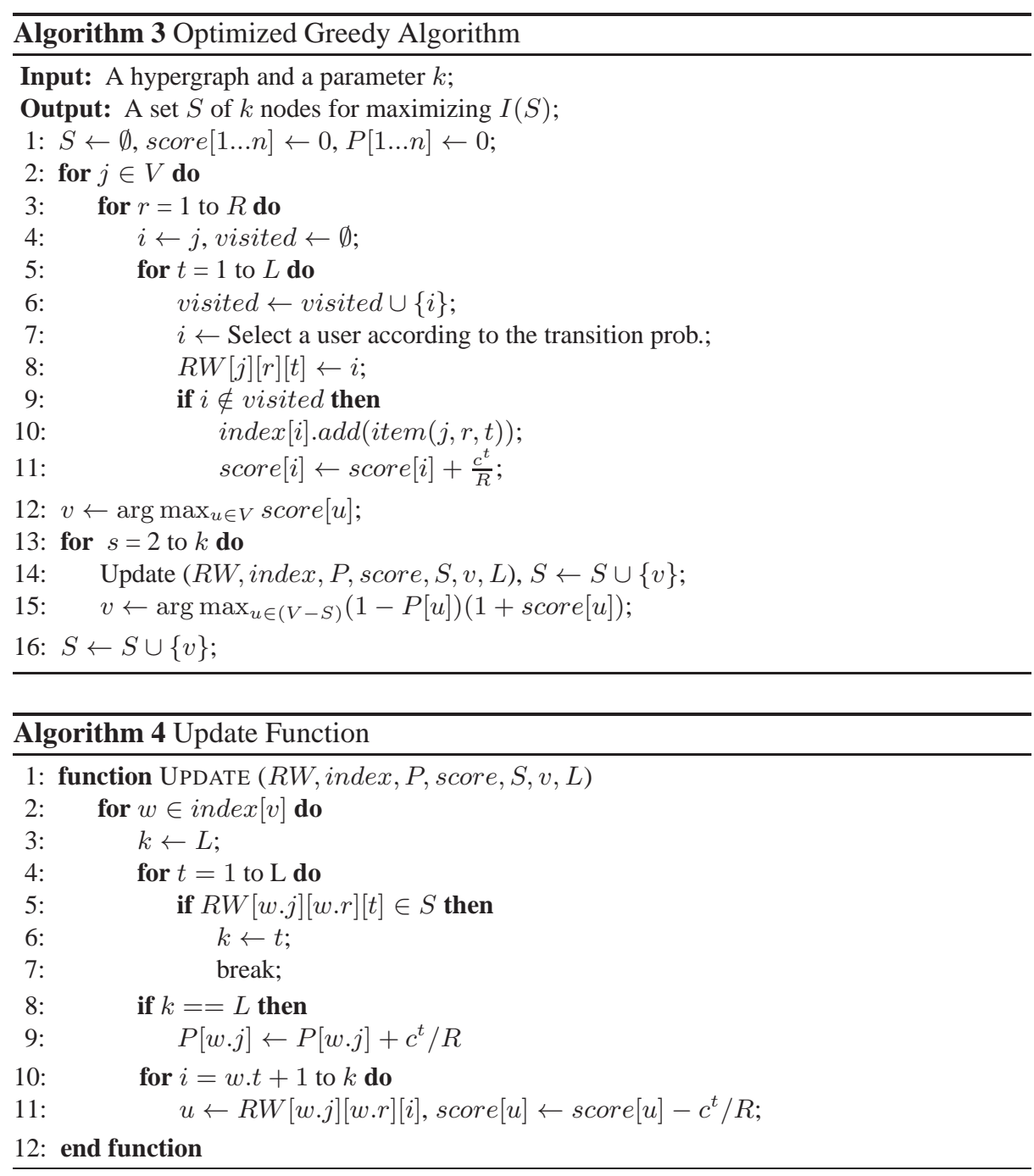

the marginal increment of all nodes, i.e., $\Delta(u)$ for every $u$, in parallel. Specifically, when performing $R$ random walks from a particular node $j$, we measure the contribution of $j$ to the marginal increment of every node. In other words, we obtain $P^{S}(j, u, h)$ for every $u$ by using only the $R$ random walks starting from $j$. As a result, we need only $O(n R)$ random walks to derive the marginal increment of all nodes, i.e., $\Delta(u)$ for every $u$, instead of $O\left(n^{2} R\right)$ random walks as in the baseline greedy algorithm.

- Walk Reuse: The core idea is that in each iteration of choosing one node to maximize the marginal increment, we record the total $O(n R)$ random walks in memory, and apply the updates accordingly after one node is added into the result set. By doing this, we can reuse the $O(n R)$ random walks to derive the marginal increment in the next iteration instead of starting new random walks from each node again. 
By incorporating the above optimization techniques, we can reduce the time complexity to $O(n R L)$, where $L$ denotes the maximum walk length. In other words, we can use the $L$ leading terms to estimate $\sum_{h=1}^{\infty} c^{h} P^{S}(j,\{u\}, h)$ and $\sum_{h=1}^{\infty} c^{h} P(u, S, h)$ as described in 4 . Thus, we let each walk runs for $L$ steps at most. Algorithm 3 states the procedure. We use score $[u]$ and $P[u]$ to record $\sum_{j \in V-S \cup\{u\}} \sum_{h=1}^{\infty} c^{h} P^{S}(j,\{u\}, h)$ and $\sum_{h=1}^{\infty} c^{h} P(u, S, h)$ for computing $\Delta(u)$, respectively. Algorithm 3 runs in two phases. The first phase (line 1-13) is to select the first seed node by running random walks and also record all the walking information for reuse. The second phase (line 1418 ) is to select the remaining $k-1$ nodes based on the stored information which requires to be updated after selecting each node. We give the update function in Algorithm 4

The update function is to update the walk information stored in score and P. Every time after we selecting a node $v$, the random walk in the following iterations should stop when it encounters $v$, and the values stored in score and $P$ should change accordingly. To achieve this, for each random walk that hits $v$ (line 2), we first check if it has visited any node in $S$ (line 4-7). If not, we increase $P[w \cdot j]$ after adding $v$ in $S$ (line 8,9). Since the following walks should stop when hitting $v$, we update score $[u]$ if node $u$ is visited after $v$ (line 10-12).

\section{Experiments}

To show the efficiency and effectiveness of our approach, we conduct experiments on real-world datasets. In particular, we first show that incorporating online activities in seed selection can lead to a significant improvement on the influence spread, i.e., influence more users with the same seed size. Then we show that our IM-RW algorithm takes much less running time than the state-of-the-art influence maximization algorithm, while achieves almost the same influence spread.

\subsection{Datasets}

We consider three datasets from social rating systems: Ciao [16], Yelp [1] and Flixster [11]. Such social rating networks are composed of a social network, where the links can be interpreted as either friendships (undirected link) or a following relationship (directed link), and a rating network, where a link represents that a user assigns a rating (or writes a review) to a product. Assigning a rating corresponds to an online activity, and multiple users assigning ratings to the same product means that they participate in the same online activity. In the rating network, we remove rating edges if the associated rating is less than 3 so as to filter out the users who dislike a product. Through this we guarantee that all the remaining users who give ratings to the same product have similar interests, e.g., they all like the product. Since the original Flixster dataset is too large to run the state-of-the-art influence maximization algorithms, we extract only a subset of the Flixster dataset for comparison studies. In particular, since the OSN of Flixster is almost a connected component, we randomly select a user, and run the breadth-first search algorithm until we get 300,000 users. We state the statistics of the three datasets in Table 1 All algorithms are run on a server with two Intel Xeon E5-2650 2.60GHz CPU and 64GB memory. 


\begin{tabular}{|c|c|c|c|c|c|}
\hline Dataset & Users & Links in OSN & Products & Ratings & OSN Type \\
\hline Ciao & 2,342 & 57,544 & 15,783 & 32,783 & directed \\
\hline Yelp & 174,100 & $2,576,179$ & 56,951 & 958,415 & directed \\
\hline Flixtser & 300,000 & $6,394,798$ & 28,262 & $2,195,134$ & undirected \\
\hline
\end{tabular}

Table 1: Datasets Statistics.

\subsection{The Benefit of Incorporating Activities}

We first show that incorporating online activities in seed selection can lead to a significant improvement on the influence spread. We fix the seed size $k$ as 50. To show the impact of activities, we use the state-of-the-art influence maximization algorithm IMM [17] to select the seed set on OSNs and use our IM-RW algorithm to select the seed set on SANs which take online activities into account. Then we use simulations to estimate the expected influence spread of the selected $k$ users on SANs and denote the results as $\sigma(\mathrm{OSN})$ and $\sigma(\mathrm{SAN})$, respectively. Finally, we define the improvement ratio on the expected influence spread as $[\sigma(\mathrm{SAN})-\sigma(\mathrm{OSN})] / \sigma(\mathrm{OSN})$.

To present the key insights, we consider the simple case in which there is only one type of users and online activities. Namely, all users have a same value of $\alpha$ which indicates the weight of activities. We emphasize that our model also works in the general case of multiple types of users and online activities.

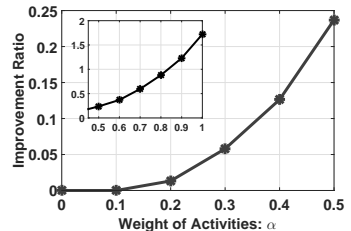

(a) Ciao

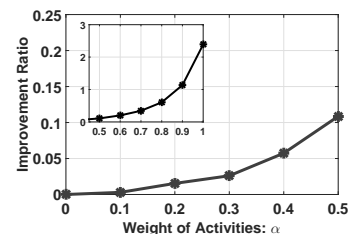

(b) Yelp

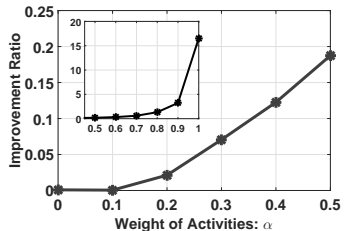

(c) Flixster

Fig. 2: Impact of online activities on influence spread.

Figure 2 depicts the improvement of incorporating online activities by varying the weight of activities $\alpha$ from 0 to 1 . The horizontal axis shows the value of $\alpha$, and the vertical axis presents the corresponding improvement ratio. From Figure 2 , one can observe that the improvement ratio is 0 when $\alpha=0$. This is because users are not affected by other users through online activities when $\alpha=0$. As $\alpha$ increases, the improvement ratio also increases. This shows that as users are more prone to be affected by other users through online activities, incorporating online activities bring larger benefit. When $\alpha=0.5$, the improvement ratio is around $25 \%$ for Ciao dataset. That is, we can influence $25 \%$ more users when incorporating online activities in the seed selection. Similar conclusions can also be observed for the datasets of Yelp and Flixster. It is interesting to observe that as $\alpha$ approaches to one, the improvement ratio reaches up to 16 for Flixster, which implies a more than an order of magnitude improvement. In summary, incorporating online activities in the seed selection by using IM-RW significantly improves the selection accuracy. 


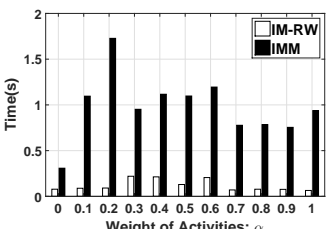

(a) Ciao

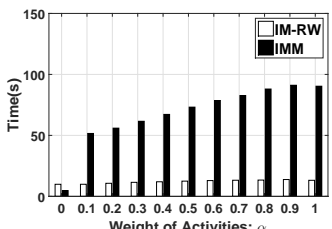

(b) Yelp

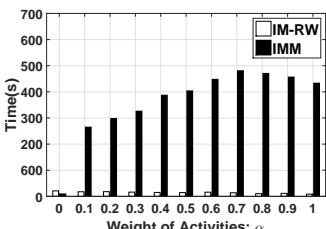

(c) Flixster

Fig. 3: Running time of IM-RW and IMM with different activity weights.

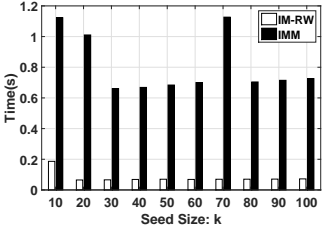

(a) Ciao

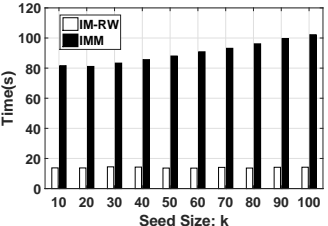

(b) Yelp

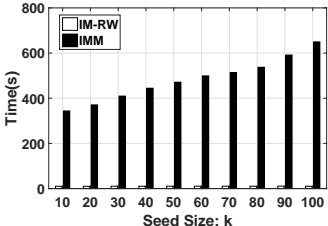

(c) Flixster

Fig. 4: Running time of IM-RW and IMM with different seed sizes.

\subsection{Performance Evaluation of IM-RW}

In this subsection, we validate the efficiency and effectiveness of IM-RW by comparing it with IMM, which is the state-of-the-art algorithm for solving influence maximization problem in OSNs, from two aspects, the running time and the influence spread. Note that IMM was originally developed for OSNs without online activities being considered, so for fair comparison, we transform SANs to a weighted graph by also taking online activities into account, and then apply IMM on the weighted graph to derive the most influential nodes.

We first compare the running time of IM-RW and IMM by varying the weight of activities $\alpha$ and the seed size $k$, and the results are presented in Figure 3 and Figure 4 Specifically, Figure 3 shows that IMM takes much longer time than IM-RW, especially when the network is large and online activities become more important (i.e., with larger $\alpha$ ). This is because as $\alpha$ increases, the time cost of IMM depends more on user-activityuser links than user-user links. Thus, as the amount of user-activity-user links is much more than that of user-user links in SANs, the time cost of IMM will increase. On the other hand, when we fix $\alpha$ as 0.8 and vary the seed size $k$, Figure 4 also shows that IMM takes much longer time than IM-RW under all settings. Therefore, we can conclude that our IM-RW algorithm really improves the efficiency of solving the influence maximization problem in SANs with online activities being considered.

We further show the influence spread of the most influential users selected by the two algorithms in Figure 5 The horizontal axis shows the values of $\alpha$, and the vertical axis represents the corresponding influence spread. We see that by taking online activities into consideration, both IMM and IM-RW can achieve almost the same performance. Because IMM is an influence maximization algorithm with theoretical performance guarantees, we can conclude that our IM-RW approach also has a good performance to maximize the influence spread.

Summary: Our IM-RW algorithm achieves a good performance in both the running time and the influence spread by taking online activities into account in SANs. In partic- 


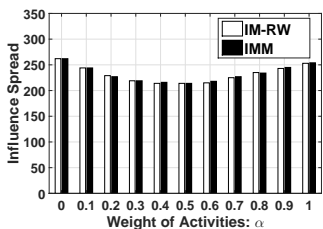

(a) Ciao

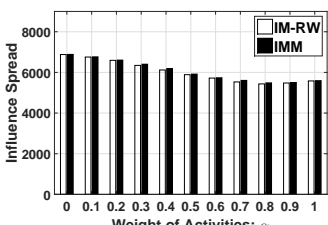

(b) Yelp

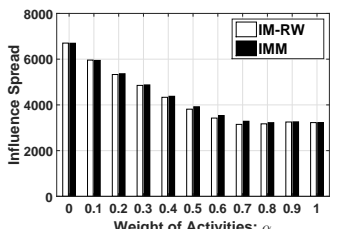

(c) Flixster

Fig. 5: Influence spread of IM-RW and IMM.

ular, comparing to the state-of-the-art algorithm IMM, our IM-RW algorithm achieves almost the same performance in seed selection, while it only requires much less running time.

\section{Related Work}

Influence maximization problem in OSNs was first formulated by Kempe et al. [12], and in this seminal work, the authors proposed the IC model and the LT model. Since then, this problem receives a lot of interests in academia in the past decade [4-6]. Because of the NP-hardness under both the IC model [4] and the LT model [6], many of the previous studies focus on how to reduce the time complexity. Recently, Borgs et al. [3] developed an algorithm which maintains the performance guarantee while reduces the time complexity significantly, and Tang et al. [17, 18] further improved the method and proposed the IMM algorithm, which is the state-of-the-art solution for influence maximization in OSNs. Besides reducing the computation overhead, several works improved the influence models, for example, topic-aware influence model [2], competitive influence model [14], opinion-based influence model [9] etc.

Centrality measure based approach was also studied, for example, the studies [5,7, 8, 19] find the most influential nodes based on degree centrality and closeness centrality. In terms of random walk, it is widely used to analyze big graphs, e.g., PageRank computation [15], graph sampling [20], and SimRank [13] etc.

We would like to emphasize that our work differs from existing studies which address the traditional influence maximization problem, while we take online activities into consideration. When we consider these online activities, only considering useruser links alone may not trigger the largest influence spread. Although we can also transform the user-activity-user links to user-user links, the underlying graph may become extremely dense so that traditional methods may not be efficient.

\section{Conclusions}

In this paper, we address the influence maximization problem in SANs with a random walk approach. Specifically, we propose a general framework to measure the influence of nodes in SANs via random walks on hypergraphs, and develop a greedy-based algorithm with two novel optimization techniques to find the top $k$ most influential nodes in SANs by using random walks. Experiments with real-world datasets show that our approach greatly improves the computation efficiency, while keeps almost the same performance in seed selection accuracy compared to IMM, the state-of-the-art algorithm. 


\section{Acknowledgements}

This work was supported by National Nature Science Foundation of China (61303048 and 61379038), and Anhui Provincial Natural Science Foundation (1508085SQF214). 


\section{Appendix}

Proof of Theorem 1: Based on the definition of $h(j, S)$ in Equation (4), we can get

$$
h(j, S)=\sum_{h=1}^{\infty} c^{h} P(j, S, h), \text { for } j \notin S,
$$

where $P(j, S, h)$ denotes the probability that a random walk starting from $j$ hits a node in $S$ at the $h$-th step. Now if we denote $p_{i S}$ as the probability that a random walk starting from $i$ hits a node in $S$ in one step, we have

$$
\begin{aligned}
h(j, S) & =\sum_{h=1}^{\infty} c^{h} P(j, S, h)=\sum_{h=1}^{\infty} c^{h} \sum_{i \notin S}\left(\boldsymbol{Q}^{h-1}\right)_{j i} p_{i S}, \\
& =\sum_{i \notin S} \sum_{h=1}^{\infty} c^{h}\left(\boldsymbol{Q}^{h-1}\right)_{i j} p_{i S}=\sum_{i \notin S} c(\boldsymbol{I}-c \boldsymbol{Q})_{j i}^{-1} p_{i S}, \\
& =c \boldsymbol{e}_{j}^{T}(\boldsymbol{I}-c \boldsymbol{Q})^{-1} \boldsymbol{Q}^{\prime} \boldsymbol{e} .
\end{aligned}
$$

Note that the largest eigenvalue of $c \boldsymbol{Q}$ is less than one, so by further expanding the expression above with an infinite series, we can rewrite $h(j, S)$ as follows.

$$
h(j, S)=c \boldsymbol{e}_{j}^{T} \boldsymbol{Q}^{\prime} \boldsymbol{e}+c^{2} \boldsymbol{e}_{j}^{T} \boldsymbol{Q} \boldsymbol{Q}^{\prime} \boldsymbol{e}+c^{3} \boldsymbol{e}_{j}^{T} \boldsymbol{Q}^{2} \boldsymbol{Q}^{\prime} \boldsymbol{e}+\cdots
$$

Proof of Theorem 2: Let $X_{1}, \ldots, X_{R}$ be $R$ independent random variables with $X_{r} \in$ $[0,1]$ for all $r=1, \cdots, R$, and set $T=\left(X_{1}+\ldots+X_{R}\right) / R$. According to Hoeffding's inequality, we have $P\{|T-E(T)|>\epsilon\} \leq 2 \exp \left(-2 \epsilon^{2} R\right)$. By applying this inequality, we have

$$
\begin{aligned}
& P\left\{\left|\hat{h}^{L}(j, S)-h^{L}(j, S)\right|>\epsilon\right\} \\
= & P\left\{\left|\sum_{t=1}^{L} \frac{c^{t}}{R} \sum_{r=1}^{R} X_{r}(t)-\sum_{t=1}^{L} c^{t} E[X(t)]\right|>\epsilon\right\}, \\
\leq & P\left\{\sum_{t=1}^{L}\left|\frac{c^{t}}{R} \sum_{r=1}^{R} X_{r}(t)-c^{t} E[X(t)]\right|>\epsilon\right\}, \\
\leq & \sum_{t=1}^{L} P\left\{\left|\frac{c^{t}}{R} \sum_{r=1}^{R} X_{r}(t)-c^{t} E[X(t)]\right|>(1-c) c^{t} \epsilon\right\}, \\
\leq & 2 L \exp \left(-2(1-c)^{2} \epsilon^{2} R\right) .
\end{aligned}
$$

Proof of Theorem 3: We first briefly introduce the vertex cover problem. An instance of vertex cover problem is specified by a graph $G(V, E)$ and an integer $k$, and asks there exists a vertex set $S \subseteq V$ such that $|S| \leq k$ and for every $(i, j) \in E,\{i, j\} \cap S \neq \emptyset$.

We now map our centrality maximization problem into an instance of the vertex cover problem by taking the same graph $G(V, E)$ and asking whether there exists a 
vertex set $S$ such that $|S| \leq k$ and $I(S) \geq(n-k) \times c+k$. We aim to show that $S$ is a vertex cover if and only if $I(S) \geq(n-k) \times c+k$. Assuming $S$ is a vertex cover. Note that $h(i, S)=1$ if $i \in S$ and $h(i, S)=c$ otherwise. Observe that for every $(i, j) \in E$, $\{i, j\} \cap S \neq \emptyset$. This implies that $I(S)=(n-k) \times c+k$. Suppose $S$ is not a vertex cover, then there should be an edge $(u, v)$ which satisfies that $\{u, v\} \cap S=\emptyset$. A random walk from $u$ passing through $v$ and at last arriving at $S$ will have length at least 2. So $h(u, S)=\sum_{j \in N(u) / v} c p_{u j} h(j, S)+c p_{u v} h(v, S)$. Due to $v \notin S, h(v, S)<1$. Thus $h(u, S)<c$, which contradicts. Therefore $S$ is a vertex cover.

Proof of Theorem 4: We first show the non-decreasing property. Note that since $h(j, S)=$ 1 if $j \in S$, so we rewrite $I(S)$ as follows.

$$
I(S)=|S|+\sum_{j \in(V-S)} h(j, S) .
$$

Now suppose that a user $u \notin S$ is added into the set $S$, then the marginal increment of the influence centrality $\Delta(u)=I(S \cup\{u\})-I(S)$ can be derived as

$$
\begin{aligned}
\Delta(u) & =\sum_{j \in V} h(j, S \cup\{u\})-\sum_{j \in V} h(j, S), \\
& =1+\sum_{j \in(V-S \cup\{u\})} h(j, S \cup\{u\})-\sum_{j \in(V-S)} h(j, S), \\
& =1-h(u, S)+\sum_{j \in(V-S \cup\{u\})}[h(j, S \cup\{u\})-h(j, S)] .
\end{aligned}
$$

According to the definition of $h(j, S)$ in Equation (12) and the random walk interpretation, we rewrite $h(j, S)$ as

$$
h(j, S)=\sum_{h=1}^{\infty} c^{h} P(j, S, h), \text { for } j \notin S,
$$

where $P(j, S, h)$ denotes the probability that a random walk starting from $j$ hits a node in $S$ at the $h$-th step for the first time. Now we can rewrite $h(j, S \cup\{u\})-h(j, S)$ as

$$
\begin{aligned}
& h(j, S \cup\{u\})-h(j, S) \\
= & \sum_{h=1}^{\infty} c^{h} P(j, S \cup\{u\}, h)-\sum_{h=1}^{\infty} c^{h} P(j, S, h) \\
= & \sum_{h=1}^{\infty} c^{h}\left[P^{\{u\}}(j, S, h)+P^{S}(j,\{u\}, h)\right]-\sum_{h=1}^{\infty} c^{h}\left[P^{\{u\}}(j, S, h)+P^{S}(j,\{u\}, h) P(u, S, h)\right] \\
= & \sum_{h=1}^{\infty} c^{h} P^{S}(j,\{u\}, h)\left[1-\sum_{h=1}^{\infty} c^{h} P(u, S, h)\right] \\
= & \sum_{h=1}^{\infty} c^{h} P^{S}(j,\{u\}, h)[1-p(u, S, h)]
\end{aligned}
$$

where $P^{T}(j, S, h)$ represents the probability that a random walk starting from $j$ hits a node in $S$ at the $h$-th step for the first time without passing any node in $T$. Therefore, 
$\Delta(u)$ can be derived as follows.

$$
\begin{aligned}
\Delta(u) & =I(S \cup\{u\})-I(S) \\
& =(1-h(u, S))\left[1+\sum_{j \in V-S \cup\{u\} h=1} \sum^{\infty} c^{h} P^{S}(j,\{u\}, h)\right] .
\end{aligned}
$$

Note that $0<c<1$ and $\sum_{h=1}^{\infty} P(u, S, h) \leq 1$, so we have $h(u, S) \leq 1$ and $1-h(u, S) \geq 0$. That is, $\Delta(u) \geq 0$, and $I(S)$ is a non-decreasing function. We now show that $I(S)$ is a submodular function. Mathematically, we only need to prove that the inequality $I(S \cup\{u\})-I(S) \geq I(T \cup\{u\})-I(T)$, for $S \subseteq T$, holds. Note that $P^{S}(j,\{u\}, h) \geq P^{T}(j,\{u\}, h)$ if $S \subseteq T$. Besides, according to the non-decreasing feature of $I(S)$, we have $h(u, S) \leq h(u, T)$. Based on these inequalities and Equation (12), we can obtain $I(S \cup\{u\})-I(S) \geq I(T \cup\{u\})-I(T)$ if $S \subseteq T$. Therefore, $I(S)$ is a submodular function.

\section{References}

1. Yelp Dataset. https://www.yelp.com/dataset_challenge/dataset

2. N. Barbieri, F. Bonchi, and G. Manco. Topic-aware social influence propagation models. In Proc. of ICDM, 2012.

3. C. Borgs, M. Brautbar, J. Chayes, and B. Lucier. Maximizing Social Influence in Nearly Optimal Time. In Proc. SODA, 2014.

4. W. Chen, C. Wang, and Y. Wang. Scalable Influence Maximization for Prevalent Viral Marketing in Large-Scale Social Networks. In Proc. ACM KDD, 2010.

5. W. Chen, Y. Wang, and S. Yang. Efficient Influence Maximization in Social Networks. In Proc. of ACM KDD, 2009.

6. W. Chen, Y. Yuan, and L. Zhang. Scalable Influence Maximization in Social Networks Under The Linear Threshold Model. In Proc. of IEEE ICDM, 2010.

7. M. G. Everett and S. P. Borgatti. The Centrality of Groups and Classes. The Journal of Math. Soc., 23(3):181-201, 1999.

8. L. C. Freeman. Centrality in Social Networks Conceptual Clarification. Social Networks, 1(3):215-239, 1979.

9. S. Galhotra, A. Arora, and S. Roy. Holistic influence maximization: Combining scalability and efficiency with opinion-aware models. arXiv preprint arXiv:1602.03110, 2016.

10. W. Hoeffding. Probability Inequalities for Sums of Bounded Random Variables. Journal of The American Statistical Association, 58(301):13-30, 1963.

11. M. Jamali and M. Ester. A Matrix Factorization Technique with Trust Propagation for Recommendation in Social Networks. In Proc. of ACM RecSys, 2010.

12. D. Kempe, J. Kleinberg, and É. Tardos. Maximizing The Spread of Influence Through a Social Network. In Proc. of ACM KDD, 2003.

13. M. Kusumoto, T. Maehara, and K.-i. Kawarabayashi. Scalable Similarity Search for Simrank. In Proc. of ACM SIGMOD, 2014.

14. Y. Lin and J. C. Lui. Analyzing Competitive Influence Maximization Problems With Partial Information: An Approximation Algorithmic Framework. Performance Evaluation, 91:187204, 2015.

15. L. Page, S. Brin, R. Motwani, and T. Winograd. The PageRank Citation Ranking: Bringing Order to The Web. Tech. report, 1999. 
16. J. Tang, H. Gao, and H. Liu. mTrust: Discerning Multi-Faceted Trust in a Connected World. In Proc. of ACM WSDM, 2012.

17. Y. Tang, Y. Shi, and X. Xiao. Influence Maximization in Near-Linear Time: A Martingale Approach. In Proc. of ACM SIGMOD, 2015.

18. Y. Tang, X. Xiao, and Y. Shi. Influence Maximization: Near-Optimal Time Complexity Meets Practical Efficiency. In Proc. of ACM SIGMOD, 2014.

19. J. Zhao, J. Lui, D. Towsley, and X. Guan. Measuring and Maximizing Group Closeness Centrality over Disk-Resident Graphs. In Proc. of SIMPLEX, 2014.

20. J. Zhao, J. Lui, D. Towsley, P. Wang, and X. Guan. A Tale of Three Graphs: Sampling Design on Hybrid Social-Affiliation Networks. In Proc. of IEEE ICDE, 2015. 